\title{
Adapting the local response for malaria elimination through evaluation of the 1-3-7 system performance in the China-Myanmar border region
}

\author{
Duoquan Wang ${ }^{1}$, Chris Cotter ${ }^{2}$, Xiaodong Sun ${ }^{3}$, Adam Bennett ${ }^{2}$, Roly D. Gosling ${ }^{2}$ and Ning Xiao ${ }^{\text {* }}$
}

\begin{abstract}
Background: Assessing the essential components of '1-3-7'strategy along the China-Myanmar border is critical to identify gaps and challenges to support evidence-based decision making.

Methods: A mixed-method retrospective study including quantitative and qualitative analysis of the 1-3-7 system components was conducted. Sampled counties were chosen based on malaria incidence from 1 January 2012 to 31 December 2014.

Results: All 260 confirmed malaria cases from sampled counties were reported within 1 day and had completed case investigations. $70.0 \%$ of all Reactive Case Detection (RACD) events were conducted and $90.1 \%$ of those were within 7 days. Only ten additional individuals were found malaria positive out of 3662 individuals tested (0.3\%) by rapid diagnostic test during RACD events.

Conclusions: Key gaps were identified in case investigation and RACD activities in Yunnan Province border counties. This evidence supports improving the RACD (or "7") response strategy in this setting. Given the challenges in this border region, it will be critical to adapt the RACD response to promote the malaria elimination along the China border.
\end{abstract}

Keywords: Malaria elimination, China-Myanmar border, 1-3-7 Strategy, Reactive case detection, Evaluation

\section{Background}

A strong political commitment was made by the National Health and Family Planning Commission of the People's Republic of China (former Ministry of Health) by issuing the National Malaria Elimination Action Plan (NMEAP; 2010-2020) in 2010. The goal of the NMEAP is to eliminate locally acquired malaria by the end of 2015, except for the bordering areas in Yunnan Province, and to interrupt local malaria transmission by 2020 nationwide [1]. The National Malaria Elimination Programme (NMEP)

\footnotetext{
*Correspondence: ningxiao116@126.com

${ }^{1}$ National Institute of Parasitic Diseases, Chinese Center for Disease Control and Prevention, Key Laboratory of Parasite and Vector Pathology, World Health Organization Collaborating Centre for Tropical Diseases, National Center for International Research on Tropical Diseases, Shanghai 200025, People's Republic of China

Full list of author information is available at the end of the article
}

strategy in China is a surveillance and response system for case reporting, investigation, and follow-up response known as '1-3-7': reporting of malaria cases within 1 day, case confirmation and investigation within 3 days, and appropriate foci response to prevent potential local transmission within 7 days [2]. The '1-3-7' strategy was rolled out nationally in 2012 and is an important and simple set of targets for local Centers for Disease Control and Prevention at the country level to follow.

Surveillance and response is the most important component driving the NMEP as the efficient response to individual cases and the foci treatment are vital strategic measures in eliminating malaria transmission [3, 4]. As malaria transmission declines, strategies for detecting and targeting clusters of infection, whether geographic or demographic, become important to reduce the local parasite reservoir [5]. Many countries are currently 
implementing a reactive case detection (RACD) strategy to achieve and maintain malaria elimination [6-10]. RACD targets spatial and temporal factors associated with finding infections and involves testing symptomatic and asymptomatic individuals residing in a specified area, typically a pre-determined radius around locally acquired or imported cases with potential transmission risk $[11,12]$.

Previous assessments of the '1-3-7' targets identified gaps in reporting in the malaria information system, particularly in Yunnan Province [13, 14]. Furthermore, recent field investigations indicated that malaria active case detection practices (including RACD) varied widely from county to county [15]. Therefore, an assessment of the '1-3-7' performance in the Yunnan Province border region was conducted to identify the gaps and challenges of the system with a focus on RACD-related activities. The ways to optimize the response strategy to the local Yunnan setting were explored to promote malaria elimination in the study areas.

\section{Methods}

Study areas

Yunnan Province-situated in the southwestern part of China with an area of $394,000 \mathrm{~km}^{2}$ and $4061 \mathrm{~km}$ of borderline-has a population of 45 million. The rural population along this border area is among the poorest in China with an average annual gross domestic product per capita of $<$ US\$ 100 [16]. Historically, Yunnan Province has stable malaria endemicity due to its mountainous valleys, proximity to the Indian Ocean and a Pacific monsoon climate along with frequent human movement contributing to the highest burden of malaria in the areas that border Myanmar. The malaria cases detected in 18 counties along the China-Myanmar border account for about 30\% of the total cases in China, with more than $95 \%$ of them in the border region being imported. The border areas face many challenges including continuous importation of malaria infections, increases in population movement and wide distribution of efficient vectors [17].

\section{Study sites}

Sampled counties were chosen based on having the highest malaria incidence in Yunnan Province from 1 January 2012 to 31 December 2014. A total of four counties (Genma, Longchuan, Tengchong, Yingjiang) out of 18 total border counties were identified with three townships randomly selected from each of the sampled counties as the study sites (Fig. 1). There are 16 health facilities (including 4 County CDCs and 12 township hospitals) in the study areas that are required to report online all suspected and laboratory-confirmed malaria cases through the China Information System for Disease Control and Prevention (CISDCP).

\section{Study design}

A mixed methods retrospective study including the quantitative and qualitative survey and analysis of the 1-3-7 system components was conducted.

\section{Study population}

Key health facility personnel from 4 local county CDCs and 12 township hospitals having at least 3 years of experiences on malaria control and elimination were selected from each sampled study area to assess the knowledge as well as practices of malaria case reporting and follow-up.

\section{Study procedures}

Questionnaires were administered to the sampled township hospitals and county CDC personnel involved in case reporting, case investigation and RACD activities. A total of 29 individuals were administered self-reported questionnaires from May to July 2015 using the standardized questionnaires with open- and closed-questions developed based on the local standard operating procedures. Questions were designed to assess the knowledge and practices of malaria case reporting, case investigation and RACD for key personnel conducting these activities.

Data on malaria case reporting, case investigation and RACD were extracted from the patients registered in 4 local CDCs and 12 sampled township hospitals for the 3-year period from 1 January 2012 to 31 December 2014. Data on malaria case reporting were extracted from the CISDCP database of the sampled counties. Data were queried and confirmed between May and December, 2015. Variables assessed in this review included the date of diagnosis, date of case reporting, date of case investigation, case classification (local vs imported), date of RACD, population tested living in areas during RACD and number of individuals tested during RACD.

\section{Quality control and assurance}

The study protocol was developed and finalized with inputs from stakeholders as well as experts. A pre-survey was conducted and the questionnaire was finalized before start of the study. Data were collected using Microsoft Excel and data were double entered. All data were summarized and verified with the sampled township hospitals and local CDCs within 15 days after completion of field survey.

\section{Data analysis}

Summary statistics and proportions were used to compare case reporting completeness, and timeliness based on the CISDCP database and original patient registers 


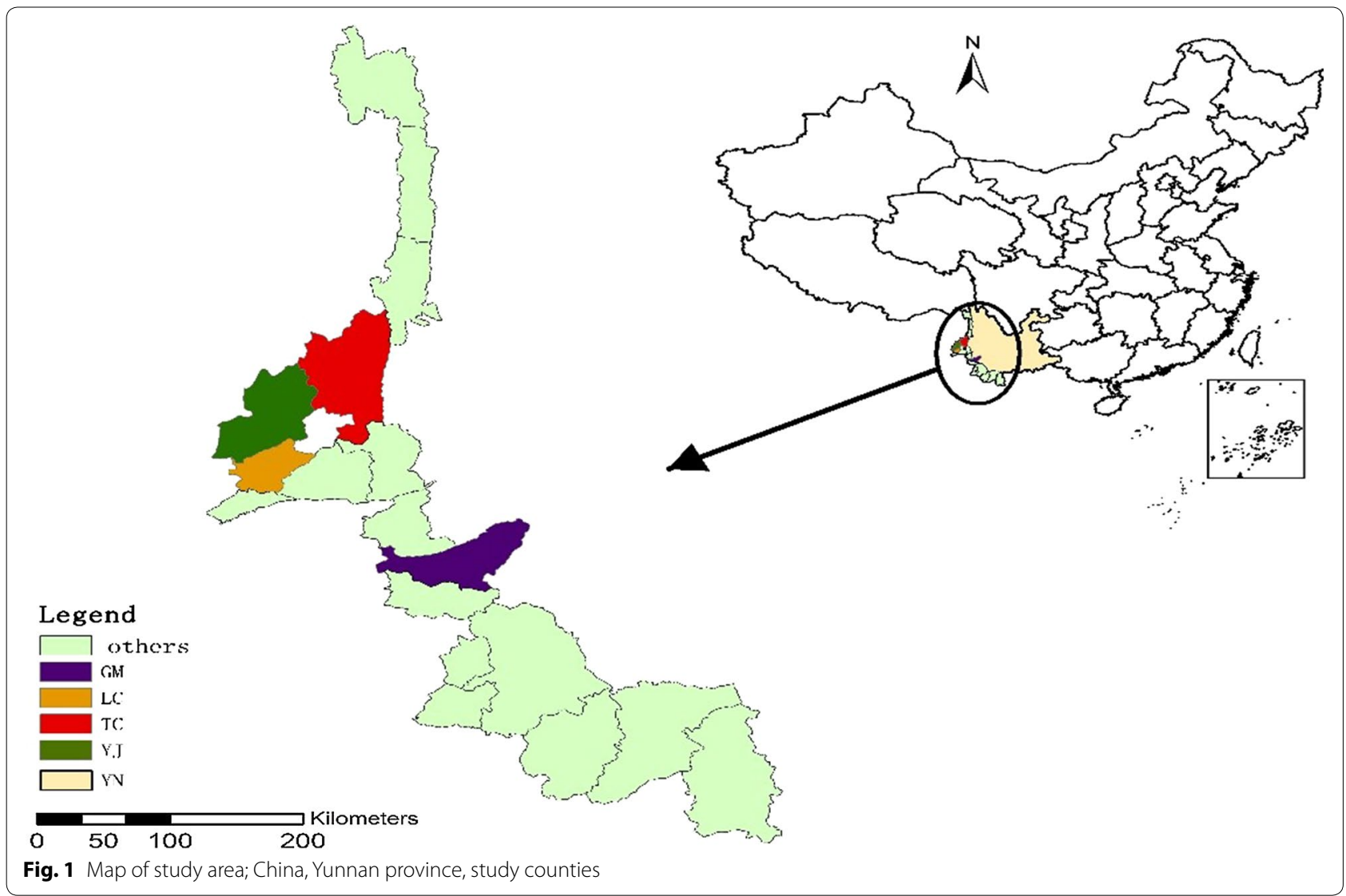

in the study areas. Case investigation and RACD screening summary statistics and proportions were based on the original patient registers among the 4 sampled counties. RACD indicators were compared between the local and imported cases, high and low transmission seasons. RACD completion rates were further analysed using Stata software, version 13.1 (Stata Corporation, College Station, TX, USA). Chi square tests $\left(\chi^{2}\right)$ were used. Confidence intervals are $95 \%$ and $P$ values are two tailed. The indicator for 'population living in areas tested' is the estimated population residing in a natural village where those individuals are at risk of contracting malaria. Malaria cases were defined based on the diagnostic criteria of World Health Organization (WHO). Annual malaria incidence was calculated by using the annual population of each county. Demographic data at the county level was obtained from the Chinese Natural Resources Database [18].

\section{Results}

A total of 29 key health facility staff were interviewed about their knowledge of case investigation and RACD activities in the study areas. Malaria case reporting, investigation and RACD indicators were assessed and results described below.

\section{Knowledge of case investigation and RACD}

Sixty-nine percent (20/29) and 65\% (19/29) of respondents correctly stated that case investigation and RACD should occur in 3 and 7 days, respectively (Table 1). 76\% $(22 / 29)$ of respondents stated that all household members (asymptomatic and fever) should be tested during RACD but only $42 \%$ (13/31) stated that testing be conducted by visiting each individual household. The knowledge of the minimum geographic radius to test around an index case household varied greatly from 50 to $100 \mathrm{~m}(38 \%, 11 / 29)$ up to $1 \mathrm{~km}(10 \%, 3 / 29)$.

\section{Summary of case reporting and investigations}

A total of 260 cases were recorded at the sampled township hospitals and county CDCs from 2012 to 2014 (Table 2). All cases were reported to the CISDCP database and within 1 day of presentation to the health facilities. All 260 cases had completed case investigations. The timeliness of case investigations conducted within 3 days was $81.5 \%(212 / 260)$ across the all sampled areas. 
Table 1 Summary of case investigation and RACD knowledge and practices

\begin{tabular}{|c|c|c|c|}
\hline \multirow[t]{2}{*}{ Question 1} & \multicolumn{3}{|c|}{ Within how many days should case investigation occur? } \\
\hline & Answer & $\mathbf{n}$ & $\%$ \\
\hline & 1 day & 2 & 7 \\
\hline & 2 days & 2 & 7 \\
\hline & 3 days & 20 & 69 \\
\hline & 7 days & 5 & 17 \\
\hline & Total & 29 & 100 \\
\hline \multirow[t]{7}{*}{ Question 2} & \multicolumn{3}{|l|}{ Within how many days should RACD occur? } \\
\hline & Answer & $\mathbf{n}$ & $\%$ \\
\hline & 1 day & 2 & 7 \\
\hline & 3 days & 6 & 21 \\
\hline & $4-5$ days & 2 & 7 \\
\hline & 7 days & 19 & 65 \\
\hline & Total & 29 & 100 \\
\hline \multirow[t]{7}{*}{ Question 3} & \multicolumn{3}{|c|}{ What is the minimum geographic radius to screen around an index case household during RACD? } \\
\hline & Answer & $\mathbf{n}$ & $\%$ \\
\hline & $<50 \mathrm{~m}$ & 8 & 28 \\
\hline & $50-100 \mathrm{~m}$ & 11 & 38 \\
\hline & $>100-500 \mathrm{~m}$ & 7 & 24 \\
\hline & $>500-1000 \mathrm{~m}$ & 3 & 10 \\
\hline & Total & 29 & 100 \\
\hline \multirow[t]{6}{*}{ Question 4} & \multicolumn{3}{|c|}{ Which individuals should you screen when conducting RACD around an index case household? } \\
\hline & Answer & n & $\%$ \\
\hline & Febrile only & 6 & 21 \\
\hline & All (asymptomatic and febrile) & 22 & 76 \\
\hline & Other & 1 & 3 \\
\hline & Total & 29 & 100 \\
\hline \multirow[t]{5}{*}{ Question 5} & \multicolumn{3}{|c|}{ How do you conduct RACD around the index case household? } \\
\hline & Answer & $\mathrm{n}$ & $\%$ \\
\hline & Visit each house and test household members & 13 & 42 \\
\hline & $\begin{array}{l}\text { Collect the community members into } \\
\text { one location and test them }\end{array}$ & 18 & 58 \\
\hline & Total & 31 & 100 \\
\hline \multirow[t]{7}{*}{ Question 6} & \multicolumn{3}{|c|}{ What is the minimum geographic radius to screen around an index case household during RACD? } \\
\hline & Answer & n & $\%$ \\
\hline & $<50 \mathrm{~m}$ & 8 & 28 \\
\hline & $50-100 \mathrm{~m}$ & 11 & 38 \\
\hline & $>100-500 \mathrm{~m}$ & 7 & 24 \\
\hline & $>500-1000 \mathrm{~m}$ & 3 & 10 \\
\hline & Total & 29 & 100 \\
\hline
\end{tabular}

\section{Summary of RACD}

\section{Screening during high and low transmission seasons}

Of the 260 malaria cases that were reported and investigated, $70.0 \%(182 / 260)$ had RACD screening conducted and $90.1 \%(164 / 182)$ of the RACD screenings occurred within 7 days (Table 3). Differences were observed in reported cases that had RACD follow-up between the high (May-October) and low (November-April) 
Table 2 Total case reporting and case investigation timeliness in study areas, 2012-2014

\begin{tabular}{|c|c|c|c|c|c|c|c|}
\hline \multirow[t]{2}{*}{ Study county } & \multirow[t]{2}{*}{ Cases recorded (\% of total) } & \multicolumn{3}{|c|}{ Cases reported } & \multicolumn{3}{|c|}{ Cases investigated } \\
\hline & & No. & Within 1 day & $\%$ & No. & Within 3 days & $\%$ \\
\hline GM & $31(11.9)$ & 31 & 31 & 100 & 31 & 24 & 77.4 \\
\hline LC & $27(10.4)$ & 27 & 27 & 100 & 27 & 24 & 88.9 \\
\hline TC & $146(56.2)$ & 146 & 146 & 100 & 146 & 118 & 80.8 \\
\hline YJ & $56(21.5)$ & 56 & 56 & 100 & 56 & 46 & 82.1 \\
\hline Total & 260 & 260 & 260 & 100 & 260 & 212 & 81.5 \\
\hline
\end{tabular}

transmission seasons $86.7 \%(130 / 150)$ and $47.3 \%$ $(52 / 110)$, respectively $\left(\chi^{2}=46.90, \mathrm{p}<0.000\right)$. RACD event follow-up within 7 days showed similar timelines with $89.2 \%(116 / 130)$ and $94.1 \%(48 / 51)$ in the high and low transmission seasons, respectively.

\section{RACD results}

Differences in RACD screening results were identified when comparing local and imported index cases in the high and low transmission seasons (Table 4). Of the 182 cases that had RACD completed, 71\% (130/182) were conducted during the high transmission seasons, with $100 \%(39 / 39)$ of local and $82 \%(91 / 111)$ of imported cases completed. In both high and low transmission seasons, $89 \%(41 / 46)$ of local cases had RACD conducted in 7 days. Follow-up of imported cases within 7 days during the high transmission seasons was $86 \%(78 / 91)$.

Of the 31,408 individuals that live in villages where the 182 RACD events took place, $11.7 \%(3662 / 31,048)$ were tested for malaria using rapid diagnostic test (RDT) or microscopy (Table 4). After local index cases were identified during high transmission seasons, 15.3\% (508/3318) were tested compared to $12.2 \%(2122 / 17,434)$ following imported index case identification. The average number of individuals tested per RACD event completed was the highest with imported index cases during high (23.3\%) and low transmission (21.1\%) seasons. All malaria-positive individuals (10 total) identified during RACD were in the high transmission seasons with $40 \%(4 / 10)$ and $60 \%$ $(6 / 10)$ from local and imported cases, respectively.

\section{Discussion}

Although study results showed that malaria case reporting was $100 \%$, critical gaps were identified in the timeliness and completeness of case investigation and RACD follow-up activities, as well as the knowledge and practices of malaria personnel conducting response activities.

Results for 2012-2014 in the study areas showed that malaria case reporting and case investigation was $100 \%$ complete. This is possible due to China enacting legislation mandating that all suspected or confirmed malaria cases are reported within 1 day of presentation for diagnosis [1]. Adherence to mandatory reporting standards is supported by a reliable web-based reporting system through the CISDCP which enables rapid reporting within 1 day. However, the timeliness of the case investigations conducted within 3 days was only $81.5 \%$, because it is very difficult for timely follow-up by County CDC staff in the remote and mountainous terrain or border areas with highly mobile populations [17, 19], and local County CDC staff may not be available to conduct the malaria case investigations due to having other responsibilities. In these circumstances, staff in county hospitals would be required to undertake the case investigations. This poses a challenge because health facility staff may not have been trained on malaria case investigations. Therefore, training of county hospital staff on case investigation and foci treatment may alleviate this challenge when County CDC staff are not available.

Study findings also indicated that there was a lack of standard operating procedures (SOPs) and, therefore, knowledge on the '1-3-7' strategy targets, highlighted in how different activities were carried out. Though a national action plan for malaria elimination is available [1], SOPs for response activities do not exist. For example, less than half of respondents (42\%) stated that RACD around an index case household should be conducted by visiting individual households rather than community screening in a single location. This may lead to unsatisfactory coverage of the targeted population, with only those who are living nearby or interested in being tested for malaria actually receiving a test. Additionally, $21 \%$ of respondents stated that they only test the symptomatic individuals during RACD events instead of all individuals residing in a pre-determined radius around a local index case. A review of Asia Pacific countries showed a wide variety of screening procedures for RACD indicating that gaps remain in the optimal methods to identify additional malaria infections during RACD [6]. However, given the importance and frequency of asymptomatic infections in low transmission settings, testing symptomatic individuals alone will not effectively reduce the residual 


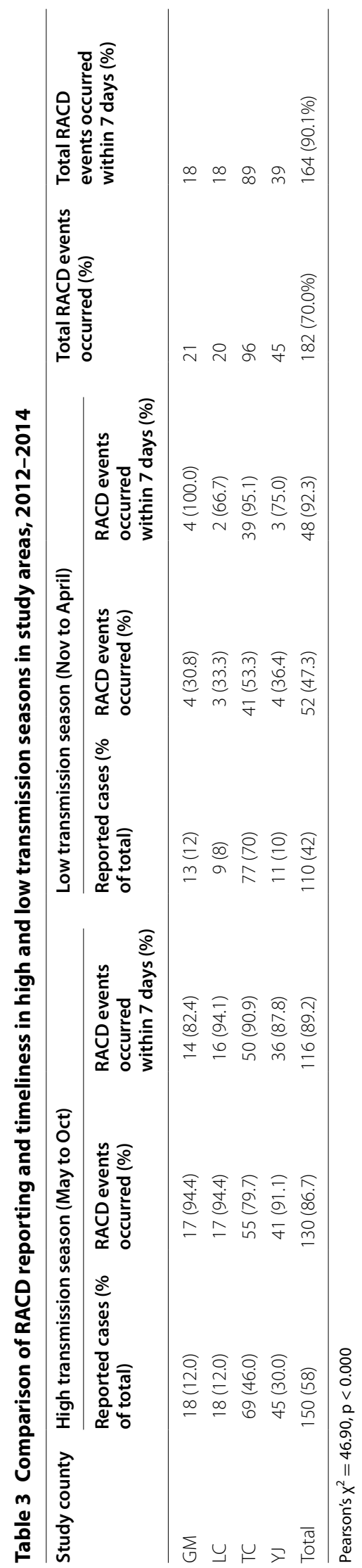




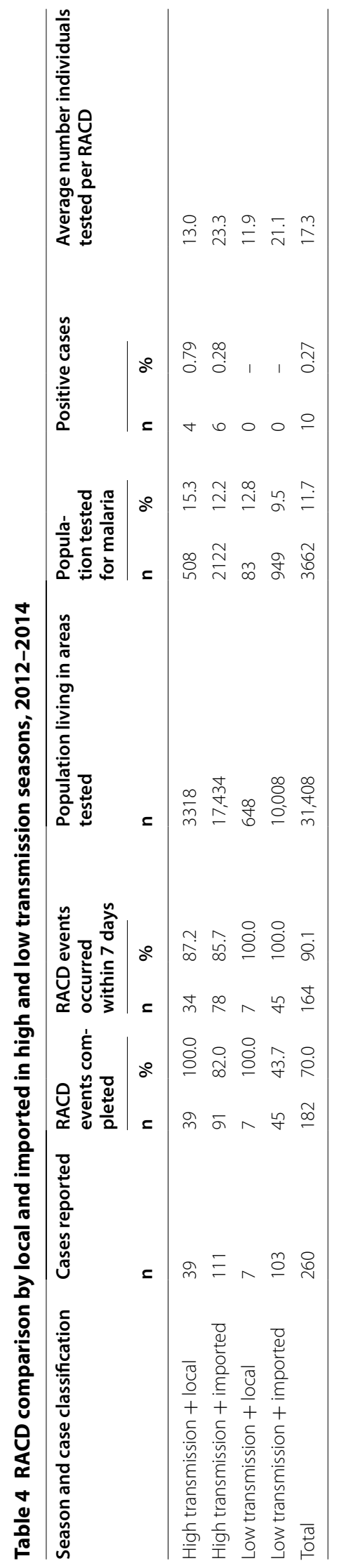


transmission [20, 21]. The NMEP should create detailed SOPs for RACD and foci response, including testing of all individuals living in an area with a recent index case to achieve higher coverage.

Although, the NMEAP describes that all imported and local index cases should have RACD completed within 7 days when transmission risk is believed to be possible [22], RACD response was lacking in both completeness and timeliness; only $70 \%$ of malaria cases had RACD follow-up conducted during the study period. In addition, more evidence is required to determine the most effective diagnostic tools for RACD screening practices in elimination settings. RACD activities in the study areas relied heavily on rapid diagnostic tests (RDTs), yielding only 10 additional positive cases. The total positivity rate of $0.27 \%$ is lower than that of national sentinel surveillance results with a positivity rate of $0.8 \%$ in 2013 [23], highlighting the inefficiency of identifying additional cases with RACD activities in the border area. Meanwhile, four infections were found from a cohort of 505 RDTs $(0.8 \%)$ and microscopy-negative people in the same areas in 2013 using CLIP-PCR.

All 10 additional infections identified in this study were in the high transmission seasons; 4 infections $(0.79 \%$ positivity) found with RACD around suspected local cases and 6 infections $(0.28 \%$ positivity) around suspected imported cases. Since only household and community members with fever were tested using RDTs during RACD, the detection of very low density infections is critically important for China in its pursuit of elimination. The availability of highly sensitive and field-friendly RDT or other molecular technique such as LAMP [23] or PCR [24] would be ideal in this border region. However, in the absence of this, presumptive treatment during the high transmission seasons targeting high-risk individuals may be a strategy to address the challenge of low density infections missed by RDTs and poor RACD screening coverage [25].

Despite China's efforts to strengthen intervention activities in the border region [26], residual malaria transmission continues to be a challenge. Recent evidence indicates that malaria importation and local vector susceptibility to imported Plasmodium vivax infections remains a continued risk along the China-Myanmar border [27]. Difficulty in classifying malaria cases as local or imported in highly mobile populations residing along the border area is also a challenge in determining the risk of resurgence from imported cases [28]. Improving the case investigation questionnaire to obtain a detailed travel history and risk behaviours may help improve case classifications as imported or local. The combination of more accurate information and developing maps for foci of transmission by geo-locating cases to the household can support the targeting of these high-risk individuals and their communities with malaria interventions [29, 30]. Furthermore, developing local capacity for genotyping of all local and imported malaria cases would be an important tool for Yunnan to determine the relatedness of malaria infections and distinguish between local and imported malaria [31].

The Yunnan border region is the ideal environment for malaria transmission. Political instability, military conflict, and a fragile health system in Myanmar, coupled with population movement along the shared border, make malaria control activities in this porous region challenging. Greater economic and health care support has been recently proposed by the Chinese government to cooperate with neighboring countries [32]. This cross-border support was also recently highlighted in the Global Technical Strategy for Malaria 2016-2030 [33] and the Strategy for Malaria Elimination in Greater Mekong Subregion (2015-2030) [34] to enable good commitment, collaboration and coordination. As the threat of multidrug resistance looms in the Greater Mekong Subregion, effective cross-border collaboration through a multi-sectoral approach is essential for both countries to support malaria elimination.

Operational research specific to this border region is also needed to support evidence-based strategies due to the unique challenges in Yunnan Province, including hard-to-reach, mobile populations, and a variety of different mosquito vectors. For example, an understanding of when to initiate the screening response after an imported case is identified-whether 7 days or possibly longer (i.e., 2-3 weeks) - is needed. By tailoring the response to this local context, available resources can be maximized to promote and accelerate malaria elimination in the border region.

Study limitations include the potential bias associated with records from public health facilities. Bias or error may exist between the log records or working reports and practices of the staff responsible for completing the reports. The authors did not explore the reasons why RACD was unable to be completed in areas within study counties; however, a deeper understanding of these reasons may help to identify ways to alleviate barriers to follow-up (Additional files 1 and 2).

\section{Conclusions}

Study results identified gaps in the knowledge and practices of RACD activities. The '1-3-7' strategy would benefit by considering malaria intervention responses adapted to the unique and challenging setting of mountainous border regions. Improving the knowledge and training of 
all staff responsible for RACD will support the standardization and implementation of response activities to promote malaria elimination in China.

\section{Additional files}

Additional file 1. Questionnaire responses.

Additional file 2. Questionnaires.

\section{Abbreviations}

RACD: reactive case detection; NMEP: National Malaria Elimination Programme; NMEAP: National Malaria Elimination Action Plan; CISDCP: China Information System for Disease Control and Prevention; CDC: Center for Disease Control and Prevention; WHO: World Health Organization; SOP: standard operating procedure; RDT: rapid diagnostic test; LAMP: loop-mediated isothermal amplification; PCR: polymerase chain reaction.

\section{Authors' contributions}

DW, CC and NX designed the study. DW and XD organized and supervised the field work. DW and CC analysed the data, drafted the manuscript and interpreted the data. NX, AB and RDG reviewed manuscript and contributed to interpretation of the data. All authors read and approved the final manuscript.

\begin{abstract}
Author details
${ }^{1}$ National Institute of Parasitic Diseases, Chinese Center for Disease Control and Prevention, Key Laboratory of Parasite and Vector Pathology, World Health Organization Collaborating Centre for Tropical Diseases, National Center for International Research on Tropical Diseases, Shanghai 200025, People's Republic of China. ${ }^{2}$ Malaria Elimination Initiative, Global Health Group, University of California, San Francisco, San Francisco, CA 94158, USA. ${ }^{3}$ Yunnan Institute of Parasitic Diseases, Puer 665000, People's Republic of China.
\end{abstract}

\section{Acknowledgements}

We would like to thank the people of the study areas for their support. Special thanks are extended to Dr. Shengguo Li for his technical assistance. We also thank the reviewers for their thoughtful comments on the manuscript.

\section{Competing interests}

The authors declare that they have no competing interests.

\section{Availability of data and materials}

The datasets supporting the conclusions of this article are available and can easily be made available upon reasonable request, when required.

\section{Consent for publication}

Not applicable. There is no presentation of confidential or identifiable data in this manuscript.

\section{Ethics approval}

Ethics approval was obtained from the National Institute of Parasitic Diseases, Chinese Center for Disease Control and Prevention (World Health Organization Collaborating Centre for Tropical Diseases, National Center for International Research on Tropical Diseases) ethical committee.

\section{Funding}

WDQ receives support from WHO/TDR (HQTDR1409931). Support for this analysis was made possible in part through a 2014 Asia Pacific Malaria Elimination Network Fellowship with support by the University of Queensland in Brisbane, Australia. Additional support was provided by the Malaria Elimination Initiative at the Global Health Group of the University of California, San Francisco. The work of the Malaria Elimination Initiative is supported by the Bill\& Melinda Gates Foundation.

Received: 23 November 2016 Accepted: 20 January 2017

Published online: 31 January 2017

\section{References}

1. Ministry of Health of China. China Malaria Elimination Action plan (2010-2020). Beijing, China; 2010.

2. Cao J, Sturrock HJ, Cotter C, Zhou S, Zhou H, Liu Y, et al. Communicating and monitoring surveillance and response activities for malaria elimination: China's "1-3-7" strategy. PLoS Med. 2014;11:e1001642.

3. WHO. Disease surveillance for malaria elimination: operational manual. Geneva: World Health Organization; 2012.

4. Ohrt C, Roberts KW, Sturrock HJ, Wegbreit J, Lee BY, Gosling RD. Information systems to support surveillance for malaria elimination. Am J Trop Med Hyg. 2015;93:145-52.

5. Bousema T, Griffin JT, Sauerwein RW, Smith DL, Churcher TS, Takken W, et al. Hitting hotspots: spatial targeting of malaria for control and elimination. PLoS Med. 2012;9:e1001165.

6. Gueye CS, Sanders KC, Galappaththy GN, Rundi C, Tobgay T, Sovannaroth $\mathrm{S}$, et al. Active case detection for malaria elimination: a survey among Asia Pacific countries. Malar J. 2013;12:358.

7. Sturrock HJ, Novotny JM, Kunene S, Dlamini S, Zulu Z, Cohen JM, et al. Reactive case detection for malaria elimination: real-life experience from an ongoing program in Swaziland. PLOS ONE. 2013;8:e63830.

8. Macauley C. Aggressive active case detection: a malaria control strategy based on the Brazilian model. Soc Sci Med. 2005;60:563-73.

9. Hustedt J, Canavati SE, Rang C, Ashton RA, Khim N, Berne L, et al. Reactive case-detection of malaria in Pailin Province, Western Cambodia: lessons from a year-long evaluation in a pre-elimination setting. Malar J. 2016;15:132.

10. Stresman GH, Kamanga A, Moono P, Hamapumbu H, Mharakurwa S, Kobayashi $T$, et al. A method of active case detection to target reservoirs of asymptomatic malaria and gametocyte carriers in a rural area in Southern Province, Zambia. Malar J. 2010;9:265.

11. Sturrock HJ, Hsiang MS, Cohen JM, Smith DL, Greenhouse B, Bousema $\mathrm{T}$, et al. Targeting asymptomatic malaria infections: active surveillance in control and elimination. PLoS Med. 2013;10:e1001467.

12. Moonen B, Cohen JM, Snow RW, Slutsker L, Drakeley C, Smith DL, et al. Operational strategies to achieve and maintain malaria elimination. Lancet. 2010;376:1592-603.

13. Zhou SS, Zhang SS, Zhang L, Rietveld AE, Ramsay AR, Zachariah R, et al. China's 1-3-7 surveillance and response strategy for malaria elimination: is case reporting, investigation and foci response happening according to plan? Infect Dis Poverty. 2015;4:55.

14. Feng J, Liu J, Feng X, Zhang L, Xiao H, Xia Z. Towards malaria elimination: monitoring and evaluation of the "1-3-7" approach at the China-Myanmar border. Am J Trop Med Hyg. 2016;95:806-10.

15. National Health and Family Planning Commission of China. Field survey report of malaria elimination process during 2011-2013 in China. Beijing, China; 2014 (unpublished).

16. Statistics Bureau of Yunnan. Yunnan statistical yearbook 2000. Beijing: China Statistics Press; 2000.

17. Xu J, Liu H. The challenges of malaria elimination in Yunnan Province, People's Republic of China. Southeast Asian J Trop Med Public Health 2012;43:819-24.

18. World Bank. Population Growth in China, 2003-2013. Geneva: World Bank; 2013. http://data.worldbank.org/country/china.

19. Yin JH, Yang MN, Zhou SS, Wang Y, Feng J, Xia ZG. Changing malaria transmission and implications in China towards National Malaria Elimination Programme between 2010 and 2012. PLoS ONE. 2013;8:e74228.

20. Lindblade KA, Steinhardt L, Samuels A, Kachur SP, Slutsker L. The silent threat: asymptomatic parasitemia and malaria transmission. Expert Rev Anti Infect Ther. 2013;11:623-39.

21. Okell LC, Bousema T, Griffin JT, Ouedraogo AL, Ghani AC, Drakeley CJ. Factors determining the occurrence of submicroscopic malaria infections and their relevance for control. Nat Commun. 2012;3:1237.

22. Chinese Centers for Disease Control and Prevention. Technical scheme of malaria elimination. Beijing, China; 2011.

23. Sirichaisinthop J, Buates S, Watanabe R, Han ET, Suktawonjaroenpon W, Krasaesub S, et al. Evaluation of loop-mediated isothermal amplification (LAMP) for malaria diagnosis in a field setting. Am J Trop Med Hyg. 2011;85:594-6.

24. Cheng Z, Wang D, Tian X, Sun Y, Sun X, Xiao N, et al. Capture and ligation probe-PCR (CLIP-PCR) for molecular screening, with application to active malaria surveillance for elimination. Clin Chem. 2015;61:821-8. 
25. Gosling RD, Okell L, Mosha J, Chandramohan D. The role of antimalarial treatment in the elimination of malaria. Clin Microbiol Infect. 2011;17:1617-23.

26. Liu P, Guo Y, Qian X, Tang S, Li Z, Chen L. China's distinctive engagement in global health. Lancet. 2014;384:793-804.

27. Wang D, Li S, Cheng Z, Xiao N, Cotter C, Hwang J, et al. Transmission risk from imported Plasmodium vivax malaria in the China-Myanmar border region. Emerg Infect Dis. 2015;21:1861-4.

28. Qian YJ, Zhang L, Xia ZG, Vong S, Yang WZ, Wang DQ, et al. Preparation for malaria resurgence in China: approach in risk assessment and rapid response. Adv Parasitol. 2014;86:267-88.

29. Townes LR, Mwandama D, Mathanga DP, Wilson ML. Elevated dry-season malaria prevalence associated with fine-scale spatial patterns of environmental risk: a case-control study of children in rural Malawi. Malar J. 2013;12:407.

30. Yukich JO, Taylor C, Eisele TP, Reithinger R, Nauhassenay H, Berhane Y, et al. Travel history and malaria infection risk in a low-transmission setting in Ethiopia: a case-control study. Malar J. 2013;12:33.
31. Greenhouse B, Smith DL. Malaria genotyping for epidemiologic surveillance. Proc Natl Acad Sci USA. 2015;112:6782-3.

32. National Development and Reform Commission. Vision and Actions on Jointly Building Silk Road Economic Belt and 21st-Century Maritime Silk Road Beijing, China: Ministry of Foreign Affairs, Ministry of Commerce of the People's Republic of China with State Council authorization, 2015, First Edition. http://www.sdpc.gov.cn/gzdt/201503/t20150330_669392. html.

33. WHO. Global technical strategy for malaria 2016-2030. Geneva: World Health Organization; 2015.

34. World Health Organization Regional Office for the Western Pacific (WPRO). Strategy for malaria elimination in the Greater Mekong Subregion (2015-2030). Geneva: World Health Organization; 2015.

\section{Submit your next manuscript to BioMed Central and we will help you at every step:}

- We accept pre-submission inquiries

- Our selector tool helps you to find the most relevant journal

- We provide round the clock customer support

- Convenient online submission

- Thorough peer review

- Inclusion in PubMed and all major indexing services

- Maximum visibility for your research

Submit your manuscript at www.biomedcentral.com/submit 